\title{
Politolinguistik und/oder Sprachkritik? \\ Das Unbehagen in und an der Deskriptivität
}

Thomas Niehr (Aachen)

\begin{abstract}
In this article it is argued that a link between the analysis of political language use and language criticism exists. Nevertheless, there is disagreement as to how far the linguistic analysis of political language use should be critical or descriptive. The author of this article argues that the different approaches of lay linguistic criticism and critical discourse analysis are not convincing from a linguistic point of view. On the other hand, it is not disputed that linguistic analysis on a purely descriptive level is impossible. Particularly with regard to the euphemistic language use of the National Socialists in Germany, a purely descriptive analysis seems to be unsatisfactory. It is argued that linguistic discourse analysis allows to combine both - linguistic description and a critical view on political communication.
\end{abstract}

\section{$1 \quad$ Einleitung}

Dass es zwischen der Sprache in der Politik und der Sprachkritik Bezugspunkte gibt, davon soll an dieser Stelle ausgegangen werden (vgl. Niehr 2011a). Wie diese Bezüge allerdings aussehen oder aussehen sollten, dazu gibt es unterschiedliche Auffassungen. Die folgenden Bemerkungen verstehen sich gleichermaßen als Standort-Problematisierung wie StandortVergewisserung. Dementsprechend sind diese Bemerkungen standpunktabhängig. Dieser Standpunkt ist weniger ein politischer als ein wissenschaftlicher. Der Autor dieser Zeilen argumentiert aus Sicht der deskriptiven Linguistik, genauer: aus Sicht der Politolinguistik und aus der Perspektive einer linguistisch fundierten Sprachkritik (vgl. Kilian/Niehr/Schiewe 2010). Der Artikel ist wie folgt gegliedert:

1. Einleitung

2. Politolinguistik

3. Sprachanalysen im Dienste der politischen Kontroverse

4. Kritische Diskursanalyse und Sprachkritik

5. Linguistische Diskursanalyse als Sprachkritik

Nach einführenden Bemerkungen zur Politolinguistik werden konkurrierende Konzeptionen vorgestellt, die sich gleichermaßen als Analyse politischer Kommunikation verstehen, allerdings eher präskriptiv-sprachkritisch als deskriptiv-linguistisch argumentieren. Abschließend 
wird die linguistische Diskursanalyse als eine Konzeption von Sprachanalyse vorgestellt, die es erlaubt, Deskriptivität und Kritik miteinander zu vereinbaren.

\section{$2 \quad$ Politolinguistik}

Die Politolinguistik - der Name wurde in einem Aufsatz von Armin Burkhardt (1996) aus dem Jahre 1996 geprägt und hat sich seitdem etabliert - versteht sich als eine linguistische Teildisziplin, die es sich zur Aufgabe gemacht hat, das Sprechen und Schreiben über die und in der Politik mit linguistischen Mitteln zu analysieren. Soweit ich sehe, haben sich die Vertreter dieser Forschungsrichtung auf einen weiten Politikbegriff verständigt, der Politik als staatliches oder auf den Staat bezogenes Handeln auffasst. Für die Politolinguistik ergibt sich daraus ein breites Forschungsfeld, das sich eben nicht auf das sprachliche Handeln von Politikern beschränkt, sondern gerade auch die Meta-Ebene - das Sprechen über eben dieses sprachliche Handeln - mit einbezieht (vgl. Niehr 2014a: 15-17).

Politolinguistische Analysen verstehen sich als der deskriptiven Linguistik zugehörig, d. h. der politische Standpunkt des jeweiligen Analysators soll bei der Analyse keine Rolle spielen, wiewohl der Analysator sicherlich (und hoffentlich) einen politischen Standpunkt hat. Ob ein Linguist also z. B. als Anhänger der Linkspartei die Wahlkampfkommunikation der CSU, der AfD oder auch als konservatives FDP-Mitglied die der Linkspartei analysiert, ist unwichtig. Wenn er oder sie seine Sache gut macht, dann können die Leser dieser Analyse die politischen Präferenzen des Analysators nicht einschätzen - so wie sie auch die politischen Präferenzen eines Tagesschau-Sprechers nicht einschätzen können. Armin Burkhardt (1996: 85) hat diese Haltung in seinem programmatischen Aufsatz einmal mit dem paradoxen Terminus der "engagierten Neutralität" umschrieben: "Damit ist gemeint: methodische Analyse und emotionslose Argumentation bei Engagement in der Sache. Denn erst das nüchterne Ergebnis wissenschaftlicher Untersuchung taugt zum Argument im öffentlichen Streit".

Eine derartige Neutralität erscheint den einen als die einzig mögliche wissenschaftliche Herangehensweise. Sie beruht auf der Überlegung, dass die jeweilige linguistische Analyse unabhängig von politischen Präferenzen durchzuführen sei. Diese Forderung nimmt auch die frühe Kritik von Linguisten wie Walther Dieckmann an der Ost-West-Sprachforschung auf: Dieckmann (1967) hatte bereits damals darauf hingewiesen, dass die entsprechenden Studien zum DDR-Deutsch immer nur dazu dienten, das linguistisch zu belegen, was die Verfasser politisch ohnehin schon zu wissen glaubten. Linguistik als Legitimation politischer Standpunkte kann aber weder Prinzipien der Wissenschaft für sich in Anspruch nehmen, noch ist eine solche Art der Linguistik ein plausibles politisches Argument.

Dieser Standpunkt der Deskriptivität aber führt seinerseits zu Schwierigkeiten, ganz abgesehen davon, dass es eine rein deskriptive oder gar objektive, voraussetzungslose Linguistik nicht geben kann. Denn auch die deskriptive Linguistik geht von Voraussetzungen aus, das haben Dieckmann (2012: 46-47), Reisgl/Warnke (2013) und Vogel (2013) unabhängig voneinander gezeigt: Varietäten sollen als gleichberechtigt gelten, die Textlinguistik macht Aussagen über die Verständlichkeit und Kohärenz von Texten, die Gesprächsanalyse ermittelt die Schwierigkeiten, die in Arzt-Patienten-Gesprächen auftreten, wir gehen von einer Maxime der Kooperativität aus. Auch die Analyse politischen Sprachgebrauchs ist von derartigen Voraussetzungen nicht ausgenommen: "eine Art Kompass zur sprachwissenschaftlichen Bewertung 
gesellschaftlicher Verhältnisse" (Vogel 2014: 92) dürfte bei den er- und aufklärenden Analysen zur politischen Kommunikation immer richtungsweisend sein. Auch wenn die Ausrichtung dieses Kompasses an Wertmaßstäben wie Demokratie, Menschenrechte, Partizipation wenig umstritten sein mögen, setzt sie eben doch Standpunkte voraus - und macht damit auch Voraussetzungen (vgl. ebd.: 94-97; Roth 2004: 38-40, 77-78). Derartige Voraussetzungen, die den Kontext einer demokratisch legitimierten Auseinandersetzung um die Ausgestaltung des Gemeinwesens voraussetzen und berücksichtigen, lassen sich aber von politischer Parteinahme kategorisch unterscheiden.

Möglicherweise kommt noch ein anderer Faktor hinzu: Während die Sprachwissenschaft die meist negativen Urteile der laienlinguistischen Sprachkritiker (häufig zu Recht) zurückweist (vgl. Kilian/Niehr/Schiewe 2010: 56-91), geht sie gleichzeitig dazu über, mit Verweis auf seine Funktionalität alles das gutzuheißen, was sich sprachgeschichtlich ergeben hat (vgl. Dieckmann 2012: 47). So wird dann unversehens aus einer Zurückweisung der Kritik an der "Verstümmelung" unserer Sprache durch SMS-Kommunikation ein Lob dieser Kommunikationsform, weil sie mit nur 160 Zeichen auskommt. Auch eine solche Betrachtungsweise ist kaum mit den Prinzipien der Deskriptivität vereinbar. Dies alles allerdings bedeutet meiner Auffassung nach allerdings keineswegs, dass Deskriptivität - verstanden als Beschreibung von Sprachdaten zum Zweck ihrer Analyse - nicht weiterhin als regulative Idee (im Kant'schen Sinne) für linguistische Analysen geeignet sei.

Abgesehen von diesem Punkt ergibt sich jedoch eine weitere Schwierigkeit. Ich möchte sie am Beispiel der Behandlung der sogenannten Euphemismen darstellen. Die klassische Definition des Euphemismus umfasst die funktionale Bestimmung der Beschönigung: "beschönigender Ersatz für ein anstößiges Wort [...] mit [...] Pejorativer Konnotation" (Bußmann 2002: 205). In der Linguistik findet man darüber hinaus die Unterscheidung zwischen verhüllenden und verschleiernden Euphemismen: Während mit Hilfe der Ersteren das Aussprechen tabuisierter Wörter - häufig aus den Bereichen Krankheit, Tod, Sexualität - vermieden wird, dienen Letztere dazu, bestimmte Sachverhalte in einer für den Sprecher besonders günstigen Weise darzustellen (vgl. Forster 2013: 107-108). Sie dienen - so könnte man deutlicher formulieren - der Täuschungsabsicht des Sprechers. Und derartige verschleiernde Euphemismen stehen natürlich im Fokus sprachkritischer Bemühungen: So finden wir in den Medien regelmäßig sprachkritische Bemerkungen über Ausdrücke wie Steuer- oder Diätenanpassungen, die doch in Wirklichkeit Steuer- oder Diätenerhöhungen seien. NRW-Umweltminister Johannes Remmel hat kürzlich in einem Interview mit der Aachener Zeitung (29.08.2014) auf die Frage, ob denn in bestimmten Gebieten weiterhin gejagt werden dürfe, den bemerkenswerten Satz gesagt: "Ich würde nicht von Jagd sprechen, sondern von Wildtiermanagement". Der Mann ist Mitglied bei den Grünen, und da kommt traditionell das Waidhandwerk nicht so gut weg. Also fühlt sich der Minister bemüßigt, dem zwangsläufig blutigen Treiben durch einen bürokratisch-abstrakten Ausdruck seine Anschaulichkeit nehmen. Es bleibt abzuwarten, wann auch die Jäger diesen Sprachgebrauch für sich entdecken und sich selbst als Wildtiermanager bezeichnen.

Nun hat die deskriptive Linguistik mit der Kategorie des Euphemismus, die ja eher aus der Rhetorik stammt, ihre Schwierigkeiten. Denn natürlich setzt die sinnvolle Rede von euphemistischem Sprachgebrauch zweierlei voraus: 
1) Die Täuschungsabsicht des Sprechers im Fall der verschleiernden Euphemismen. Diese aber kann mit linguistischen Mitteln nur in Einzelfällen ex post nachgewiesen werden: Möglicherweise ist ein Politiker, der von Diätenanpassung spricht, ja tatsächlich der Meinung, dass die Diäten den gestiegenen Lebenshaltungskosten lediglich angepasst werden, ohne dass es dabei zu einer realen Erhöhung der Kaufkraft kommt. Vor diesem Hintergrund scheint seine Wortwahl kaum zu kritisieren (vgl. Roth 2004: 20-21).

2) Wenn ich beschönigenden Sprachgebrauch kritisiere, präsupponiere ich, dass es nicht-beschönigenden Sprachgebrauch nicht nur gibt, sondern dass ich ihn auch identifizieren kann. Das widerspricht aber allem, was doch die meisten Linguisten seit dem linguistic turn immer wieder betonen: die wirklichkeitskonstitutive Rolle unserer Sprache. In Bezug auf Euphemismen hat Heringer (1982: 16) dieses Problem einmal deutlich auf den Punkt gebracht:

Schwierig bleibt aber auch hier der Nachweis, daß es sich um einen Euphemismus handelt. Der Anhänger der Abbildtheorie muß sich einen privilegierten Zugang zur Realität und zur Sprache zuschreiben, damit er erkennt, daß solche Wörter [wie Preiskorrektur, Freistellung, Befriedung usw., Th. N.] nur die Realität verkleiden oder gar inhaltsleer sind. Ist Verteidigungsminister ein Euphemismus oder ist diese Benennung eine berechtigte Selbstdarstellung und Absichtserklärung oder gar der Realität angemessen?

(Heringer 1982: 16)

Man könnte es nun dabei bewenden lassen und Euphemismus zu einer Nicht-Kategorie der Linguistik erklären, ähnlich wie beispielsweise Sprecherabsicht. Damit aber handelt man sich ein weiteres Problem ein. Dies scheint mir deutlich, wenn man sich nicht auf Wörter wie Diätenanpassung und Entsorgungspark konzentriert. Betrachtet man nämlich Wörter wie Sonderbehandlung oder Euthanasie im Sprachgebrauch der Nationalsozialisten, dann wird auch der hartgesottenste Deskriptivist ein Unbehagen dabei verspüren, diese Wörter nicht als zynische Euphemismen zu bezeichnen.

Spätestens hier möchte man als Linguist über die Deskriptivität hinausgehen und - auf linguistischer Grundlage - dem gesunden Menschenverstand zu seinem Recht verhelfen. $\mathrm{Ob}$ und in welcher Weise dies möglich ist, darüber herrscht allerdings keine Einigkeit. Kersten Roth (2004: 40) hat in seiner Dissertation dargelegt, dass es einer "konstruktive[n] Verbindung" von Linguistik und Sprachkritik bedürfe, um gesellschaftlich relevante Aussagen treffen zu können. Dabei komme der Linguistik die deskriptiv-sprachwissenschaftliche Analyse zu. Die Sprachkritik hingegen sei normative Sprachreflexion. Die spezifische Kombination beider könne Grundlage einer "kooperativ-kritischen Sprachwissenschaft" bzw. beratender Politolinguistik sein (ebd.: 80-81). An der Trennung von Linguistik und Sprachkritik hält er jedoch fest. Einen anderen Standpunkt vertritt in dieser Frage beispielsweise Nina Janich (2013: 363): Sie bewertet das Festhalten an dieser Trennung als "Rückschritt hinter die Erkenntnisse der Pragmatik". Denn die Bewertung von Sprachhandlungen sei Teil des menschlichen Sprachhandlungsvermögens und damit auch Teil der Sprachreflexion (vgl. ebd.: 365). Dieser Streit soll und kann hier nicht entschieden werden. Es soll allerdings anhand von Beispielen gezeigt werden, auf welch unterschiedliche Weise bislang kritische Momente in die Analyse des politischen Sprachgebrauchs einbezogen worden sind. 


\section{Sprach-Analysen im Dienste der politischen Kontroverse}

Auch lange nach der Kritik von Dieckmann an methodisch unzulänglichen Analysen und nach der Weiterentwicklung der Politolinguistik wurde und wird immer wieder versucht, Sprach-Analysen, die im Zusammenhang politischer Kontroversen funktionalisiert werden, als Linguistik zu verkaufen. Dies sind also Analysen, die vor dem Hintergrund des bisher Gesagten viel Kritisches enthalten, von einer linguistischen Analyse kann allerdings kaum die Rede sein. Derartige Sprachbewertungen zeichnen sich häufig durch einen einseitig kritischen Blick aus, der direkte Rückschlüsse auf die politischen Sym- und Antipathien der Verfasser erlaubt. Gerne werden derartige Studien mit aufklärerischem Gestus präsentiert - gerade so als schützten die Verfasser ihre Leser vor der Manipulation der jeweiligen politischen Gegner. Zur Illustration präsentiere ich zwei Zitate. Diese sind zwar schon älteren Datums, sie wurden aber wegen ihrer Prägnanz ausgewählt. Erstens lesen wir in einem Schlagwörterbuch aus dem Jahr 1980 unter dem Stichwort Sozial:

Am sichersten fährt man heute jedoch mit der Annahme, daß es beim Sozialen vor allem ums Geld geht. Die Sozial-Schwachen sind solche, die wenig Geld haben. Die sozialen Hilfen laufen darauf hinaus, daß auch die Sozial-Schwachen mehr Geld bekommen. Die sozialen Errungenschaften besagten im Grunde nur, daß man für weniger Arbeit mehr Geld als früher bekommt. Die soziale Belastung dadurch bekommen die anderen dann am Staatssäckel indirekt als Steuerzahler und Unternehmer zu spüren.

(Traugott 1980: 107)

Und natürlich gibt es diese Art von politisch motivierter Sprachanalyse auch aus dem andern politischen Lager. So lesen wir - zweitens - bei Schau fünf Jahre später:

Von welcher Qualität die "Neusprache" der CDU ist, hat ihr Chefideologe, Heiner Geißler, mehrmals deutlich gemacht. Ihre Sprache lebt von "Begriffsbesetzungen" [...]: alte Begriffe werden beibehalten, aber ihre ursprüngliche Bedeutung wird ihnen ausgetrieben und eine neue unterlegt. In Betracht kommen für solche "Begriffsbesetzungen" jene zentralen Begriffe, die auch heute noch im gesellschaftlichen Leben und in der Politik eine große Rolle spielen und die ursprünglich einer aufklärerisch-demokratischen Tradition entstammen, auf die sich linke Politiker stützen.

Die "Solidarität" beispielsweise ist ein solcher Begriff [...]. Dieser [...] große politische Wert "Solidarität" verkommt im CDU-Grundsatzprogramm von 1978 zu einer "persönlichen Dienstleistung", wird umstilisiert und als "Zuwendung von Mensch zu Mensch" privatisiert [...]. Der "Solidarität" wird der politische Stachel gezogen, ein generelles Umwertungsverfahren, das die CDU mit den klassischen politischen Grundwerten vornimmt - zum Zweck ihrer Entpolitisierung.

(Schau 1985: 13-14)

Und die Politiker selbst haben für sich auch schon entdeckt, dass man solche Sprachanalysen als politisches Argument nutzen kann. So erklärt uns beispielsweise Oskar Lafontaine in einer Sendung von Anne Will, dass im Wort Kommunismus das lateinische communis stecke. Kommunismus wird auf diese Weise umgedeutet zu 'Gemeinschaftseigentum', einer Idee des Urchristentums, wie Lafontaine im gleichen Atemzug erklärt (vgl. Niehr 2011b).

Natürlich führen solche Analysen nicht weiter. Sie sind nicht deskriptiv, sie sind nicht wissenschaftlich. Sie sind standortgebundene Analysen, denen das Mäntelchen der Wissenschaft mit Hilfe linguistischer Versatzstücke umgehängt wird. Auf diese Weise kann das Unbehagen an der Deskriptivität sicherlich nicht beseitigt werden. 


\section{$4 \quad$ Kritische Diskursanalyse und Sprachkritik}

Aus Sicht der deskriptiven Linguistik ebenfalls problematisch sind zahlreiche Analysen der sogenannten Kritischen Diskursanalyse, und zwar aus mehreren Gründen. Einerseits sind dies methodische Gründe, die Dinge wie die Korpuszusammensetzung betreffen. Diese diskursanalytischen Interna sollen hier nicht näher beleuchtet werden (vgl. Spitzmüller/Warnke: 108-109; Niehr 2014b: 56-57). Am Beispiel der Duisburger Spielart Kritischer Diskursanalyse möchte ich aber auf das Ziel eingehen, nicht bei der Deskription der Diskurse stehenzubleiben, sondern Diskursteilnehmer zu werden, z. B. indem bestimmte Missstände angeprangert werden. Mit den Worten Siegfried Jägers (2005: 67):

Kritische Diskursanalyse beschränkt sich also nicht auf die Analyse von Sprache. Da Kritische Diskursanalyse den Diskurs zum Gegenstand hat, richtet sich ihre Kritik eben auf diesen Diskurs, der sich als Fluß von Wissen durch die Zeit darstellt, wobei dieses Wissen im wesentlichen (wenn auch nicht nur) in Text und Rede auftritt, die natürlich aus Wörtern und Sätzen bestehen, so daß sich eine solche Kritik auch auf diese diskursiven Elemente beziehen kann und muß, etwa auf Euphemismen, Argumentationsformen, Anspielungen, Redensarten, allgemeine stilistische Besonderheiten usw.

(Jäger 2005: 67; Hervorhebung im Original)

Weiterhin behauptet Jäger (2005: 69), dass die von ihm favorisierte Spielart der Diskursanalyse in der Lage sei, "Vorschläge zur Vermeidung herrschender Mißstände zu entwickeln", indem sie nicht nur vor euphemistischem und sensationslüsternem Sprachgebrauch warnt, nicht nur Sprachkritik, sondern Gesellschaftskritik betreibt, und angesichts der hochgehaltenen Normen von Demokratie, Gerechtigkeit und allgemeinen Menschenrechten geradezu dazu zwingt, Position zu beziehen, sei es gegen Krieg, gegen Rassismus, gegen Ausgrenzungen aller Art, gegen ökologische Fehlentwicklungen oder gegen die Anhäufung von Reichtum auf Kosten sozial ohnedies "schwacher" Bevölkerungsteile und vieles mehr.

Der Unterschied zur Linguistik wird in diesem Zitat deutlich markiert: Aufgrund des Deskriptionsgebots und aufgrund der Überzeugung, dass Wirklichkeit sprachlich konstituiert ist, würden deskriptive Linguisten Kategorien wie die hier zitierten nicht verwenden. Sie würden auch nicht auf die Idee kommen, mithilfe linguistischer Mittel "ökologische Fehlentwicklungen" - wie auch immer man sie definieren mag - zu bekämpfen. Offensichtlich ist also, dass einer solchen Ausrichtung Kritischer Diskursanalyse eine ideologische Haltung zugrunde liegt, aufgrund derer die jeweiligen Analysen ausgerichtet werden, nämlich "gegen" bestimmte Zustände, die als politisch bekämpfenswert angesehen werden.

Möchte man eine solche Variante der Kritischen Diskursanalyse im Kontinuum zwischen Sprachwissenschaft und Sprachkritik verorten, dann kann man feststellen, dass sie deutlich näher an der Sprachkritik anzusiedeln ist. Denn Sprachkritik bewertet Sprache oder Sprachgebrauch. Sie kann nur unter der Voraussetzung sinnvoll betrieben werden, dass man das Kritisierte auch anders ausdrücken kann - sie setzt also sprachliche Wahlmöglichkeiten voraus. Gleichzeitig nimmt sie für sich in Anspruch, die zur Wahl stehenden Alternativen aufgrund bestimmter Kriterien zu bewerten. Laienlinguistische Sprachkritik legt häufig die Kriterien richtig/falsch zugrunde. Insbesondere bei Bewertungen, die grammatische Phänomene betreffen, wird dann häufig der Eindruck erweckt, es gebe genau eine richtige und eine oder mehrere falsche Varianten. Die Beispiele einer solchen besserwisserischen Sprachkritik sind hinrei- 
chend bekannt: Es wird auf die semantischen Unterschiede von dasselbe/das Gleiche hingewiesen oder darauf, dass anscheinend und scheinbar nicht synonym seien. Im Bereich der Grammatik wird auf Deklinationsfehler (des Autors statt des Autoren) oder Syntaxfehler hingewiesen (weil mit Hauptsatzstellung). Dass eine solche oberlehrerhafte Art der Sprachkritik nicht hilfreich ist, darauf haben Linguisten, die sich für Sprachkritik interessieren, immer wieder hingewiesen (vgl. Kilian/Niehr/Schiewe 2010: 56-91; zusammenfassend Kilian/Niehr/Schiewe 2013: 301-302). Im Wesentlichen krankt eine solche Sprachkritik ja daran, dass sie konsequent Situationskontexte ausblendet und auf der einfachen und zweifelsohne unzulänglichen Richtig-falsch-Dichotomie beharrt.

Eine derartige Sprachkritik finden wir in übertragener Weise auch im Bereich des Politischen: Sie bedient sich häufig einer realistischen Diktion und fordert für sich den bereits zitierten privilegierten Zugang zur Realität ein. Sie blendet die Appellfunktion politischer Sprache aus und fokussiert sich auf die Darstellungsfunktion. Prototypisch kommt diese Art der Sprachkritik in folgendem Zitat aus den 70er Jahren zum Ausdruck:

Worte sind dazu da, Dinge zu bezeichnen. Sie sollen sagen, was ist; und sofern ihnen das gelingt, sagen sie die Wahrheit.

(Kuhn 1975: 11)

Sofern ihnen das nicht gelingt - so impliziert dieses Zitat - lügen sie. Als Linguisten wissen wir, dass es sich nicht ganz so einfach verhält, wie der Autor dieser Zeilen uns glauben machen will. Ich will auf diese Art der Sprachkritik, die natürlich ihrerseits zu politischen Zwecken eingesetzt wird, deshalb auch gar nicht weiter eingehen, sondern anhand von Beispielen doch noch einmal die Berechtigung von Kritik an politischem Sprachgebrauch verdeutlichen (vgl. Niehr 2011a). Wenn politisch interessierte Leser die folgende Wortliste sehen, dann können sie damit gleich eine sprachkritische Diskussion der BRD verbinden, die sich nicht der schlichten Argumentation laienlinguistischer Sprachkritiker bedient:

- Ratten und Schmeißfliegen (Franz Josef Strauß)

- durchrasste Gesellschaft (Edmund Stoiber)

- Tätervolk (Ex-MdB Martin Hohmann)

- gleichgeschaltete Medien (Eva Herman)

- Moralkeule (Martin Walser)

- Faszinosum (Philipp Jenninger)

- entartete Kultur (Joachim Kardinal Meißner)

- Kopftuchmädchen (Thilo Sarrazin)

In den Diskussionen, die sich an die Verwendung dieser Vokabeln entzündeten, ging es zwar einerseits um Sprachkritik, aber um eine Art von Sprachkritik, die mittels der Kritik an einem speziellen Ausdruck gleichzeitig die in diesem Ausdruck kondensierte politische Argumentation zum Ziel hatte.

Diese Art von Sprachkritik ist auch von Interesse für die Politolinguistik, weil sich hier über die Bewertung sprachlicher Ausdrücke (als mehr oder weniger angemessen) politische Stand- 
punkte artikulieren, die häufig Gegenstand gesellschaftlicher Kontroversen sind. $\mathrm{Ob}$ und in welcher Weise die Linguistik hier etwas beitragen kann, soll in den folgenden Überlegungen dargestellt werden.

\section{$5 \quad$ Linguistische Diskursanalyse als Sprachkritik}

Gehe ich vom Standpunkt der deskriptiven Linguistik aus, dann verbieten sich Parteinahmen, die im Gewand normativer Sprachregelungen daherkommen. Es wäre aus dieser Sicht also keine Lösung, einen der hier genannten Ausdrücke pauschal als "unangemessen" zu etikettieren und auf diese Weise Position zu beziehen, ganz unabhängig davon, wie man sich in der jeweiligen politischen Debatte positioniert. Mit anderen Worten: Die deskriptive Linguistik taugt wenig dazu, gegen gesellschaftliche oder politische Fehlentwicklungen Stellung zu beziehen. Dies liegt daran, dass die Linguistik kein Instrumentarium dafür hat, solche Fehlentwicklungen zu konstatieren. Das heißt nun aber keineswegs, dass die Linguistik blind bleiben muss für Phänomene der sprachlichen Ungleichbehandlung oder gar des sprachlichen Rassismus. Ich bin weiterhin der Meinung, dass sich auch die Linguistik sprachkritisch betätigen kann, allerdings sollte diese Art der Sprachkritik linguistisch und nicht politisch motiviert sein. Deshalb stellt sich zunächst die Frage, aufgrund welcher Kriterien und aufgrund welcher Daten eine linguistische Sprachkritik möglich wird. Ich beginne mit den Daten: Linguistische Sprachkritik, wenn sie denn die Namen verdienen soll, muss sich stets auf konkrete Daten beziehen, entweder auf einen empirisch erhobenen Sprachgebrauch oder auf geltende Sprachnormen, die ihrerseits über den Sprachgebrauch zu ermitteln sind. Für unseren Zusammenhang heißt dies: Allgemeine Aussagen über "die Sprache der Politiker" sind solange wertlos, wie sie sich nicht auf linguistische Textanalysen stützen, die typische Sprachmuster zu Tage fördern. Zugrunde liegen muss solchen Analysen also immer ein konkretes Sprachhandeln. Um den Unterschied zu laienlinguistischer Sprachkritik zu verdeutlichen, könnte man auch so formulieren: Vor der Bewertung hat jeweils die Analyse zu stehen, die sich auf konkrete Sprachhandlungen konkreter Sprecher/Schreiber beziehen muss. Und im Unterschied zur skizzierten Spielart der Kritischen Diskursanalyse bedarf es jeweils eines ausreichend umfangreichen Textkorpus und nicht nur einiger weniger vorgeblich repräsentativer Texte, um zu belastbaren Aussagen über den Diskurs zu kommen (vgl. dazu das Beispiel in Niehr 2014b: 57).

Die erste Aufgabe einer linguistischen Sprachkritik ist mithin die der Deskription. Dazu bedient man sich etwa diskurslinguistischer Herangehensweisen, die - verkürzt gesprochen aufzeigen, welche Akteure in welchen gesellschaftlichen Situationen über welche Dinge und Personen in welcher Weise sprechen. Derartige Analysen können ebenfalls darüber Auskunft geben, welche Redeweisen umstritten sind und zu Kontroversen führen. Und sie können diese Kontroversen im Hinblick auf ihre Argumentation analysieren. Diskursanalysen avant la lettre liegen mit Brisante Wörter (Strauß/Haß/Haras 1989) und Kontroverse Begriffe (Stötzel/Wengeler 1995) vor. Das Methodenspektrum wurde seitdem vielfältig erweitert (vgl. Niehr 2014b: 66-126) und immer wieder an konkreten Analysen zum öffentlichen Sprachgebrauch erprobt. Gegen den von Seiten der Kritischen Diskursanalyse erhobenen Einwand, dies sei lediglich die altbekannte Begriffsgeschichte, die den Namen Diskursanalyse gar nicht verdiene (vgl. Jäger 2009: 11) lässt sich mit einigem Recht behaupten, dass hier Kritik durch Deskription (vgl. Schiewe 2003: 133; Wengeler 2011: 42-44, 2014: 30-32) geübt

ISSN 1615-3014 
werde, ohne jedoch selbst einen bestimmten politischen Standpunkt einnehmen zu müssen. Eine solche Art der Kritik hat m. E. einige entscheidende Vorteile:

Ihr Ziel ist es, mit linguistischen Mitteln offenzulegen, "welche Sicht der Wirklichkeit von wem aus welchen Gründen konstituiert worden ist" (Schiewe/Wengeler 2005: 7). Das Kritische linguistischer Sprachkritik besteht darin, aufklärerisch zu wirken. Eine derartige Aufklärung, die nicht Normen setzt, sondern Normen reflektiert, kann als "Korrektiv im Machtspiel der Sprechweisen, der Diskurse" wirken (ebd.: 7). Der Vorteil einer solchen Herangehensweise liegt darin, dass die Argumentation auf einer Meta-Ebene erfolgt, also gerade nicht in der jeweiligen Kontroverse explizit Stellung bezieht. So setzt sie sich nicht dem Vorwurf aus, linguistische Urteile aufgrund ideologischer Vorannahmen zu treffen oder, um das Urteil Dieckmanns aus den 60er Jahren noch einmal aufzugreifen: Eine solche Art der Kritik setzt sich nicht dem Vorwurf aus, mit linguistischen Mitteln noch einmal das zu zeigen, was sie aufgrund ihrer politischen Einstellung ohnehin schon zu wissen glaubte.

Ich möchte diese Art einer linguistisch fundierten Sprachkritik abschließend an wenigen Beispielen illustrieren (vgl. zum Folgenden Niehr 2014a: 84-87, 106-112). In den Medien wurden beispielsweise die Rede Philipp Jenningers (1988) zum Gedenken an die Reichspogromnacht und die Rede Martin Walsers (1998) zur Verleihung des Friedenspreises des Deutschen Buchhandels ausgiebig kritisiert. Man kann vielleicht sogar von politischen Skandalen sprechen. Dies gilt ebenso für die Diskussion um die Verhaltensweisen des ehemaligen Bundespräsidenten Christian Wulff. Zu allen diesen Fällen kann eine linguistisch fundierte Sprachkritik etwas beitragen, ohne selbst politisch Stellung zu beziehen. In den ersten beiden Fällen sind es Grundprinzipien der Textlinguistik, aufgrund derer man deutlich machen kann, dass Jenninger wie auch Walser sich eines problematischen Sprachgebrauchs bedient haben. Während Jenninger die Prinzipien einer Gedenkrede eklatant verletzt hat, hat Walser statt einer öffentlichen, politischen eine an anderen Maßstäben orientierte Rede gehalten - möglicherweise waren die Maßstäbe literarische, wie er selbst in der sich anschließenden Walser-BubisDebatte mehrfach rechtfertigend als Argument angeführt hat. "Präziser kann man den Sinn öffentlicher Rede nicht mit dem der literarischen verwechseln", hat diese Vorgehensweise Josef Kopperschmidt (1999: 88) kommentiert.

Anders wiederum verhält es sich bei Wulff. Bei ihm kann man über die Verletzung der Kommunikationsmaximen nach Grice zeigen, warum sich seine Glaubwürdigkeit in der Öffentlichkeit schließlich dem Nullpunkt näherte, auch wenn sein Verhalten strafrechtlich - wie wir inzwischen wissen - kaum zu beanstanden war.

Das übergeordnete Prinzip, das derartigen linguistisch fundierten sprachkritischen Analysen zugrunde liegt, ist das rhetorisch begründbare Prinzip der funktionalen Angemessenheit. Mit ihm gehen wir davon aus, dass es nicht "den" guten, angemessenen, schlechten oder unangemessenen Sprachgebrauch schlechthin gibt. Dieser ist jeweils nur in Abhängigkeit von der Sache, der Situation und dem Publikum zu bestimmen, und zwar auf allen sprachlichen Ebenen. Vor diesem Hintergrund ließe sich beispielsweise nicht sinnvoll dafür argumentieren, dass die Verwendung bestimmter Wörter per se zu kritisieren sei: Möchte beispielsweise ein Rechtsextremist seine Gesinnung sprachlich öffentlich kundtun, dann ist die Verwendung eines Ausdrucks wie Überfremdung oder das Äußern einer Parole wie Wer betrügt, der fliegt, die von der rechtsextremen Partei ProNRW in Wahlkämpfen plakatiert wurde, sozusagen das 
Mittel der Wahl und eine sprachlich angemessene Handlung. Dass diese Handlung anderen Prinzipien zuwiderlaufen kann - etwa gesetzlichen Bestimmungen, die etwas zur Menschenwürde oder zur Gleichbehandlung aller Menschen vor dem Gesetz aussagen -, kann ebenfalls mit linguistischen Mitteln nachgewiesen werden.

Etwas ausführlicher möchte ich abschließend auf die Wulff-Affäre eingehen, um zu demonstrieren, dass auch eine deskriptiv-linguistisch fundierte Herangehensweise zu Ergebnissen kommen kann, die nicht vorwiegend durch Realitätsferne gekennzeichnet sind.

Der ehemalige Bundespräsident Wulff war zunächst in Misskredit geraten, weil er - so berichteten die Medien - einen für ihn besonders vorteilhaften Privatkredit für sein Eigenheim in Hannover zwar in Anspruch genommen, dies jedoch zunächst bestritten hatte. Hinzu kamen weitere Medienberichte über Vorteilsnahmen von Oktoberfestbesuchen, über FlugUpgrades bis hin zu Urlaubsaufenthalten, die von vermögenden Bekannten Wulffs beglichen wurden. Auch wenn Wulff für dies alles strafrechtlich nicht zu belangen war, lohnt doch ein Blick auf sein kommunikatives Verhalten, das wohl mitverantwortlich für den Vertrauensverlust und schließlich auch den unausweichlichen Rücktritt Wulffs gewesen sein dürfte.

Der Nachrichtensender n-tv hat im Verlaufe der Affäre die Frage, ob Wulff in Bezug auf seinen Hauskredit gelogen habe, wie folgt kommentiert:

Die Zeitung [Bild, Th. N.] wirft dem Staatsoberhaupt vor, während seiner Zeit als niedersächsischer Ministerpräsident 2010 falsche Angaben im Landtag von Hannover gemacht zu haben. Hintergrund ist eine Anfrage von zwei Landtagsabgeordneten der Grünen, ob Wulff zu dem Unternehmer Egon Geerkens aus Osnabrück oder dem damaligen Air-Berlin-Chef Joachim Hunold geschäftliche Beziehungen unterhalten habe. Daraufhin ließ Wulff am 18. Februar 2010 im Landtag erklären: "Zwischen Ministerpräsident Wulff und den in der Anfrage genannten Personen und Gesellschaften hat es in den letzten zehn Jahren keine geschäftlichen Beziehungen gegeben."

Klare Frage - klare Antwort

"Diese Angaben sind und waren korrekt", bestätigte erneut Wulffs Sprecher Olaf Glaeseker in Berlin. Der damalige niedersächsische Regierungschef habe zu Recht auf eine entsprechende Abgeordneten-Anfrage geantwortet, dass er keine geschäftlichen Beziehungen zu Herrn Geerkens habe. Dementsprechend wurde die unmissverständliche Anfrage "wahrheitsgemäß verneint", heißt es in der Erklärung. Tatsächlich haben sich Christian Wulff und seine Frau Bettina den 500.000-Euro-Kredit auch nicht von Herrn Geerkens, sondern von dessen Frau Edith besorgt.

(n-tv; zit. nach Niehr 2014a: 84)

Dass es sich beim Kredit von Geerkens Ehefrau sehr wohl um eine relevante Information handelte, hat Wulff später selbst zugegeben:

Bundespräsident Christian Wulff hat es bedauert, den Privatkredit über 500.000 Euro bislang verschwiegen zu haben. "Ich erkenne an, dass hier ein falscher Eindruck entstehen konnte. Ich bedauere das", teilte Wulff in einer Erklärung mit. "Es wäre besser gewesen, wenn ich auf die Anfrage der niedersächsischen Abgeordneten im Landtag über die konkreten Fragen hinaus auch diesen privaten Vertrag mit Frau Geerkens erwähnt hätte, denn in der Sache hatte und habe ich nichts zu verbergen."

(Zeit.de, zit. nach Niehr 2014a: 85)

Nun kann man an diesem Beispiel sehr schön zeigen, dass Wulff sich hier eines Verstoßes gegen die Grice'schen Maximen schuldig gemacht hat, indem er die 2. Maxime, die Quanti- 
tätsmaxime, eklatant verletzt hat: Mache Deinen Beitrag so informativ wie (für die gegebenen Gesprächszwecke) nötig. Und zwar hat er diese Verletzung wohl in der Hoffnung begangen, auf diese Weise den privaten Kredit vertuschen zu können.

Daraus und aus der Tatsache, dass solche Informationsdefizite vom jeweiligen politischen Gegner und in der Öffentlichkeit gerne mit Lügen gleichgesetzt werden, lässt sich der entstandene Vertrauensverlust gut erklären. Dies ist in etwa die Erklärung, die ich in meiner Einführung in die Politolinguistik (vgl. Niehr 2014a: 84-87) gegeben habe. Doch damit hatte die Causa Wulff noch nicht ihr Ende gefunden. Denn Wulff (2014) hat am 21.07.2014 dem SPIEGEL ein Interview gegeben und einen Monat vorher ein Buch veröffentlicht, das die Affäre aus seiner Sicht noch einmal aufarbeitet. Und darin äußert er sich auch noch einmal zu den Umständen seiner damaligen Erklärung. Bezieht man diese Äußerungen Wulffs in die Analyse mit ein, so ergibt sich möglicherweise ein geändertes Bild. Wulff macht nämlich darauf aufmerksam, dass er seinerzeit im Niedersächsischen Landtag nach anderen als den Grice'schen Kommunikationsmaximen gehandelt habe:

Die Opposition stellt hunderte von bohrenden Fragen, das ist die Aufgabe der Opposition. Regierungsmitglieder nerven diese Fragen, aber genaue Antworten sind so notwendig wie genaue Fragen. [...] Alles und jedes darf gefragt werden, und es muss wahrheitsgemäß auf alles und jedes geantwortet werden, das sind zwei gute Grundsätze. Aber es gibt in den Landesparlamenten ebenso wie im Bundestag einen Konsens, den alle anerkennen: genau das zu beantworten, was konkret gefragt wurde. Das ist Brauch. Es zwingt die Opposition, eine Frage ordentlich und präzise zu formulieren, und die Regierung, umfassend und konkret zu antworten. [...] Über eine Frage hinauszugehen und sie interpretierend auszuleuchten - die Opposition meinte sicher nicht nur Herrn Geerkens, sondern auch Frau Geerkens, sicher nicht nur geschäftliche Beziehungen, sondern auch private -, wäre der Weg in die Endlosschleife. [...] Es war nach Egon Geerkens gefragt, der keinerlei Gewerbe mehr unterhielt. Wäre die Opposition mit meiner Antwort nicht zufrieden gewesen, hätte sie eine Woche später nach Frau Geerkens fragen können. Hat sie aber nicht. Das Argument, dass die Frage so gemeint war, zählt nicht. Gemeint ist, was gefragt wird.

(Wulff 2014: 202-203)

Möchte man diese Ausführungen Wulffs in linguistische Terminologie übersetzen, dann kann man das etwa folgendermaßen tun: In bestimmten politischen Auseinandersetzungen gelten die Grice'schen Kommunikationsmaximen, deren Einhaltung wir bei unserem Gegenüber ja immer schon voraussetzen, keineswegs. Und das wissen auch alle Beteiligten. Deshalb ist es scheinheilig - so fahre ich interpretierend fort - im Nachhinein die Verletzung eben dieser Maximen zu beklagen.

Bei aller nachvollziehbaren Kritik an Wulffs Kommunikationsverhalten scheint mir dies doch ein bedenkenswertes Argument zu sein. Dies ändert freilich nichts an der Tatsache, dass sein Verhalten Wulff nur kurzfristig genützt hat. Er selbst schreibt dazu in seinem Buch: "Aus heutiger Sicht wäre es besser gewesen, die Anfrage offensiv anzugehen und den privaten Finanzierungskredit darzulegen." (ebd.: 203) "Aus heutiger Sicht" ist wohl auch das verklausulierte Eingeständnis, dass für einen Bundespräsidenten und auch für weniger prominente Personen des öffentlichen Lebens andere Kommunikationsstandards gelten als für einen Angeklagten vor Gericht. Dass dies gleichermaßen für die Verhaltensstandards gilt, sei hier nur am Rande erwähnt.

Im Hinblick auf Wulffs Argumentation kann eine linguistische Analyse auch noch an einem anderen Punkt Aufschluss geben: Wulff betont sowohl in seinem Buch wie in dem bereits 
angesprochenen Spiegel-Interview, dass er ein unbequemer Bundespräsident gewesen sei. Dies sei entscheidend dafür gewesen, dass er von den Medien zum Rücktritt gezwungen worden sei:

Ich war eine Provokation. Ich war einigen mächtigen Medienschaffenden zu unbequem geworden. Den einen war meine Einlassung - auf dem Höhepunkt der Sarrazin-Debatte -, dass der Islam inzwischen zu Deutschland gehört, ein Ärgernis. Andere verübelten mir meine Kritik an den Banken, wieder andere empörten sich über meine Kritik an der katholischen Kirche. Da kam eins zum anderen, und an einem bestimmten Punkt entstand der Eindruck, Wulff ist von Springer zum Abschuss freigegeben. Da wollten viele schlicht nicht abseitsstehen. [...] Ich spreche nicht [...] von einer Kampagne oder einem Komplott. Aber dass hier eine Art Meinungskartell am Werk war, ist ja nicht erst mir aufgefallen, eine gewisse Parallelität der Berichterstattung war vor meinem Rücktritt offensichtlich. Das hat nichts mit einer Verschwörungstheorie zu tun.

(Spiegel 21.07.2014: 18-19)

Diese Sicht Wulffs wird auch in seinem Buch deutlich, in dessen zweitem Teil die Kapitelüberschriften lauten: "Die Jagd", "Die letzte Kugel" und "Das Recht" (vgl. Wulff 2014: Inhaltsverzeichnis).

Auch eine solche Argumentation könnte mit linguistischen Mitteln ohne Weiteres überprüft werden: Die Diskursanalyse bietet die entsprechenden Instrumentarien, um beispielsweise zu überprüfen, inwieweit die von Wulff kritisierten Medien tatsächlich unisono eine Argumentationslinie vertreten haben und inwieweit die von Wulff angesprochenen Themen (Islam, Kritik an Banken und der Kirche) tatsächlich als entscheidende Argumente gegen ihn angeführt wurden. Und sie kann auch eine vom Spiegel in den Raum gestellte Frage beantworten. Auf Wulffs Darstellung kontert der Spiegel nämlich:

Ist die allgemeine Wahrnehmung der Bürger nicht eine ganz andere? Die Menschen sagen: Gut, dass Wulff freigesprochen wurde, wenn an den strafrechtlichen Vorwürfen nichts dran ist. Sie sagen aber auch: Gut, dass Wulff nicht mehr Bundespräsident ist, denn wir wollen keinen Schnäppchenjäger als Staatsoberhaupt.

(Spiegel 21.07.2014: 19)

Woher der Spiegel diese "allgemeine Wahrnehmung der Bürger" zu kennen glaubt, bleibt sein Geheimnis. Eine linguistische Analyse von Internet-Kommentaren, Leserbriefen und anderen öffentlich zugänglichen Texten "der Menschen" jedenfalls könnte derartigen Behauptungen eine solide Grundlage geben. Eine solche Analyse könnte darüber hinaus kritisch werden, wenn sie zeigte, dass in der Debatte um den Wulff-Rücktritt je nach Bedarf die Begründungssprachen gewechselt werden. Dass nämlich eine Beurteilung des Falles Wulff unter moralischer Perspektive zu anderen Ergebnissen kommt als unter rechtlicher Perspektive, wäre anhand der im Diskurs verwendeten Argumente ebenfalls aufzuzeigen.

Für derartige Analysen bedarf es einer Herangehensweise, die das Methodenspektrum der Linguistik anzuwenden weiß. Dieses bietet kritisches Potenzial in Fülle. Eines zusätzlichen, ideologisch zu begründenden Standpunkts bedarf es dazu meiner Auffassung nach nicht.

\section{Literatur}

Burkhardt, Armin (1996): "Politolinguistik. Versuch einer Ortsbestimmung". In: Klein, Josef/Diekmannshenke, Hajo (Hrsg.): Sprachstrategien und Dialogblockaden. Linguistische 
und politikwissenschaftliche Studien zur politischen Kommunikation. Berlin/New York, de Gruyter: 75-100.

Bußmann, Hadumod (Hrsg.) (2002): Lexikon der Sprachwissenschaft. 3. aktualisierte und erweiterte Auflage. Stuttgart: Kröner.

Dieckmann, Walther (1967): "Kritische Bemerkungen zum sprachlichen Ost-West-Problem." Zeitschrift für deutsche Sprache 23: 136-165.

Dieckmann, Walther (2012): Wege und Abwege der Sprachkritik. Bremen: Hempen.

Forster, Iris (2013): "Euphemismen im lebenslangen Lernen und politischen Handeln". In: Kilian, Jörg/Niehr, Thomas (Hrsg.): Politik als sprachlich gebundenes Wissen. Politische Sprache im lebenslangen Lernen und politischen Handeln. Bremen, Hempen: 107-118.

Heringer, Hans Jürgen (1982): "Sprachkritik - die Fortsetzung der Politik mit besseren Mitteln". In: Heringer, Hans Jürgen (Hrsg.): Holzfeuer im hölzernen Ofen. Aufsätze zur politischen Sprachkritik. Tübingen, Narr: 3-34.

Jäger, Siegfried (2005): "Diskurs als 'Fluß von Wissen durch die Zeit'. Ein transdisziplinäres politisches Konzept". Aptum. Zeitschrift für Sprachkritik und Sprachkultur 1: 52-72.

Jäger, Siegfried (2012): Kritische Diskursanalyse. Eine Einführung. 6. vollständig überarbeitete Auflage. Münster: Unrast.

Janich, Nina (2013): "Sprachreflexion als Mittel der Aufklärung und Sprachkultivierung: von der Sprachkritik zur Sprecherkritik". Mitteilungen des Deutschen Germanistenverbandes 60/4: 356-373.

Kilian, Jörg/Niehr, Thomas/Schiewe Jürgen (2010): Sprachkritik. Ansätze und Methoden der kritischen Sprachbetrachtung. Berlin/New York: de Gruyter.

Kilian, Jörg/Niehr, Thomas/Schiewe, Jürgen (2013): "Es gibt kein Falsches im Angemessenen. Überlegungen zu einem sprachkritischen Analysemodell". Mitteilungen des Deutschen Germanistenverbandes 60/4: 300-320.

Kuhn, Helmut (1975): "Despotie der Wörter. Wie man mit Sprache die Freiheit überwältigen kann". In: Kaltenbrunner, Gerd-Klaus (Hrsg.): Sprache und Herrschaft. Die umfunktionierten Wörter. Freiburg/Basel/Wien, Herder: 11-19.

Niehr, Thomas (2011a): "Politische Sprache und Sprachkritik". Mitteilungen des Deutschen Germanistenverbandes 3: 278-288.

Niehr, Thomas (2011b): "Neoliberalismus oder Kommunismus? Über eine pseudolinguistische Variante der Begriffsbesetzung". Sprachreport 3: 2-7.

Niehr, Thomas (2014a): Einführung in die Politolinguistik. Göttingen/Bristol: Vandenhoeck \& Ruprecht.

Niehr, Thomas (2014b): Einführung in die linguistische Diskursanalyse. Darmstadt: Wissenschaftliche Buchgesellschaft.

Reisigl, Martin/Warnke, Ingo H. (2013): "Diskurslinguistik im Spannungsfeld von Deskription, Präskription und Kritik. Eine Einleitung". In: Meinhof, Ulrike Hanna/Reisigl, Martin/Warnke, Ingo H. (Hrsg.): Diskurslinguistik im Spannungsfeld von Deskription und Kritik. Berlin, Akademie-Verlag: 7-36.

Roth, Kersten Sven (2004): Politische Sprachberatung als Symbiose von Linguistik und Sprachkritik. Zu Theorie und Praxis einer kooperativ-kritischen Sprachwissenschaft. Tübingen: Niemeyer. 
Schau, Albrecht (1985): Von AWACS bis Zwangsanleihe. ABC aktueller Schlagwörter. Göttingen: Steidl.

Schiewe, Jürgen (2003): "Wege der Sprachkritik nach 1945". In: Wengeler, Martin (Hrsg.): Deutsche Sprachgeschichte nach 1945. Hildesheim/Zürich/New York, Olms: 125-138. (= Germanistische Linguistik 169-170).

Traugott, Edgar (1980): Schlagwörterbuch für Bürger und Zeitungsleser. Entzauberte Parolen von A(Aggression) bis z(zeitgemäss). Freiburg/Basel/Wien: Herder.

Vogel, Friedemann (2013): "Linguistische Diskursanalyse als engagierte Wissenschaft?! Ein Plädoyer für eine 'Theorie der Praxis als Praxis'". In: Meinhof, Ulrike Hanna/Reisigl, Martin/Warnke, Ingo H. (Hrsg.): Diskurslinguistik im Spannungsfeld von Deskription und Kritik. Berlin, Akademie-Verlag: 279-298.

Vogel, Friedemann (2014): "Linguistik als Kampfsport - Ein Plädoyer für die Suche nach Paradigmen demokratischen Sprechens in Alltag, Medien und Recht". Linguistik online 69/7: 91-108. doi: 10.13092/10.69.1658.

Wengeler, Martin (2011): "Linguistische Diskursanalysen - deskriptiv, kritisch oder kritisch durch Deskription?" In: Schiewe, Jürgen (Hrsg.): Sprachkritik und Sprachkultur. Konzepte und Impulse für Wissenschaft und Öffentlichkeit. Bremen, Hempen: 35-48.

Wengeler, Martin (2014): "Sprachwissenschaft - nur wissenschaftlich oder auch kritisch?" In: Niehr, Thomas (Hrsg.): Sprachwissenschaft und Sprachkritik. Perspektiven ihrer Vermittlung. Bremen, Hempen: 27-37.

Wulff, Christian (2014): Ganz oben - ganz unten. München: Beck. 